\title{
Hadron multiplicities at COMPASS
}

\author{
Nicolas DU FRESNE VON HOHENESCHE* ${ }^{* \dagger}$ \\ On behalf of the COMPASS collaboration \\ Johannes Gutenberg-Universtät, Institut für Kernphysik, Johan-Johannes-Becher Weg 45, 55128 \\ Mainz, Germany, E-mail: dufresnedcern.ch
}

Quark fragmentation functions (FF) $D_{q}^{h}\left(z, Q^{2}\right)$ describe final-state hadronisation of quarks $q$ into hadrons $h$. The FFs can be extracted from hadron multiplicities produced in semi-inclusive deep inelastic scattering. The COMPASS collaboration has recently measured charged hadron multiplicities for identified pions and kaons using a $160 \mathrm{GeV} / \mathrm{c}$ muon beam impinging on an isoscalar LiD target. The data cover a large kinematical range and provide an important input for global QCD analyses of world data at NLO, aiming at the determination of FFs. The latest results from COMPASS on pion multiplicities and pion fragmentation functions will be discussed.

XXII. International Workshop on Deep-Inelastic Scattering and Related Subjects 28 April - 2 May 2014

Warsaw, Poland

\footnotetext{
${ }^{*}$ Speaker.

${ }^{\dagger}$ Supported by BMBF
} 


\section{Introduction}

The spin structure of the nucleon is an interesting topic within particle physics. Acquiring more knowledge about the hadronisation process will help to investigated the flavour separated spin structure. Semi-inclusive deep inelastic scattering can be factorised within the QCD parton model [1] into three components, the hard scattering cross section, the parton distribution functions (PDF), and the fragmentation functions (FF). The PDFs describe the structure of the initial-state hadrons while the FFs describe the hadronisation process as a collinear conversion of a single parton into final-state hadrons. In semi-inclusive deep inelastic scattering (SIDIS), flavour separated fragmentation functions can be determined in leading order from measured hadron multiplicities $M^{h}\left(Q^{2}, x, z\right)$, i. e. from the numbers of final-state hadrons divided by the number of observed DIS events, using:

$$
M^{h}\left(x, Q^{2}, z\right)=\frac{1}{\sigma^{D I S}} \frac{d \sigma^{h}}{d x d z d Q^{2}}=\frac{\sum_{q} e_{q}^{2} q\left(x, Q^{2}\right) D_{q}^{h}\left(z, Q^{2}\right)}{\sum_{q} e_{q}^{2} q\left(x, Q^{2}\right)}
$$

Here, $q\left(Q^{2}, x\right)$ are the parton distribution functions of the struck quarks, $e_{q}$ the charge of the quark with the flavour $q$ and $D_{q}^{h}\left(z, Q^{2}\right)$ the fragmentation function of quark $q$ into hadron $h$. The kinematic variables are the negative virtual photon four-momentum squared $Q^{2}$, the Bjorken variable $x$ and the hadron energy fraction $z$. The current interest in FFs is motivated, $i . g$. by contradicting results of the strange quark helicity density. The contribution of the strange quarks to the nucleon spin, $\Delta s+$ $\Delta \bar{s}$, can be extracted from an inclusive measurement [2] of the polarised structure function $g_{1}\left(x, Q^{2}\right)$ for proton and deuteron using $\mathrm{pQCD}$. The result in NLO is negative. On the other hand, $\Delta s(x)$ can be extracted using semi-inclusive hadron asymmetries [3] in combination with a parametrisation of parton distribution functions and fragmentation functions. The integral over the measured $x$ range tends to be zero, but strongly depends on the used parametrisation (i.g. EMC [4] and DSS [5]). The COMPASS experiment is a well-suited facility to study fragmentation functions from semi-inclusive muon nucleon scattering in a large kinematic range.

\section{The COMPASS experiment}

The COMPASS experiment [6] is located at the M2 beam line of the Super Proton Synchrotron (SPS) at CERN. It uses a tertiary muon beam impinging on an isoscalar fixed-target. The set-up consists of a beam spectrometer, a target and two magnetic spectrometer stages. Both magnetic spectrometer stages have high resolution trackers and hadron as well as electromagnetic calorimetry. The first spectrometer stage also includes a Ring Imaging CHerenkov (RICH) detector for particle identification, mainly the separation of pions and kaons.

\section{Multiplicities}

COMPASS recorded data with a $160 \mathrm{GeV}$ muon beam scattering off a polarised lithiumdeuterid $\left({ }^{6} \mathrm{LiD}\right)$ target in 2006. The direction of the polarisation of the target was regulary reversed, so that the polarisation cancels out. After quality checks and geometric cuts on the interaction vertex of the beam muon and the target nucleon, events are selected within the DIS range with the 


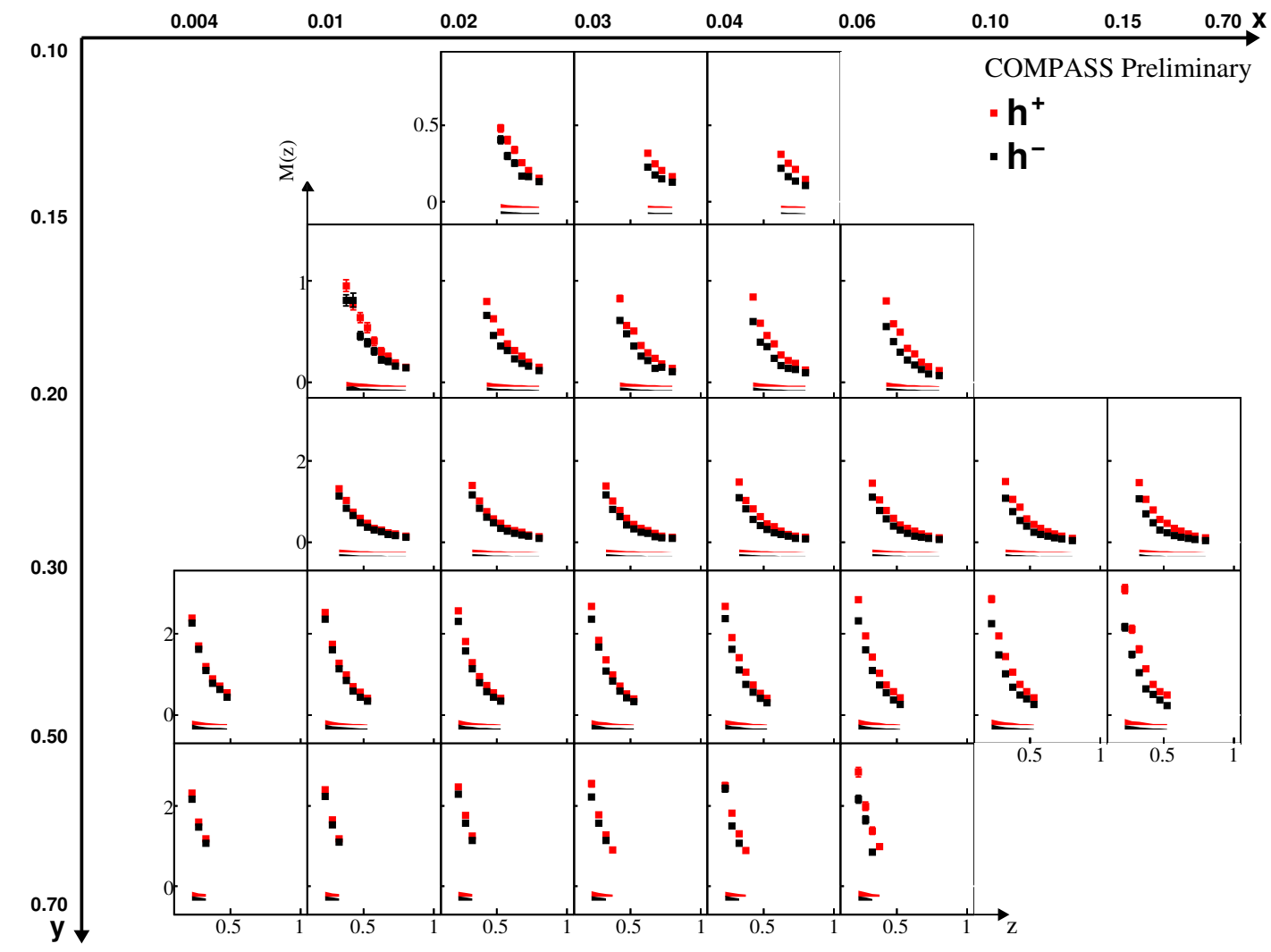

Figure 1: The preliminary, corrected unidentified hadron multiplicities in bins of $x, y$ and $z$ for positive hadrons (red) and negative hadrons (black). The systematic uncertainties are shown as error bands.

following cuts: $Q^{2}>1 \mathrm{GeV}^{2}, 5<W<17 \mathrm{GeV}$ for the invariant mass of the final hadronic state, $0.004<x<0.7$ and $0.1<y<0.7$ for the relative virtual photon energy. The hadrons are constrained to $0.2<z<0.85$. The lower energy cut is introduced to suppress contributions from target fragmentation and the upper limit to reduce contributions from production of exclusive vector mesons. A hadron momentum cut of $10 \mathrm{GeV}<P_{h}<40 \mathrm{GeV}$ has been applied to assure a fair separation between pions and kaon. A three-dimensional binning of $x, y$ and $z$ is used in the analysis. The obtained raw multiplicities are corrected for the acceptance of the spectrometer, the particle identification efficiency and for radiative QED effects.

The acceptance has been determined with a Monte Carlo simulation of the COMPASS experiment. It includes the LEPTO [7] generator which contains the parton distribution functions MSTW08 and the JETSET [8] package for the hadronisation model according to the Lund string model. Here, the COMPASS tuning [9] is used. The produced events are reconstructed in a GEANT3 model of the COMPASS experiment. The three dimensional acceptance correction is smooth in $z$ and reaches values from 0.45 to 0.7 . The systematic error is estimated to be $5 \%$. Due to the hadron momentum cut some extrapolation using LEPTO into the non-measured range is necessary. All kinematic bins where the extrapolation contribution is higher than $10 \%$ are excluded to avoid model dependence. The RICH efficiency is determined by using the detector with the final-states of well-known particles $\left(K_{S}^{0}, \phi\right.$ and $\left.\Lambda\right)$. Depending on the hadron momentum and the entrance angle in the detector, 


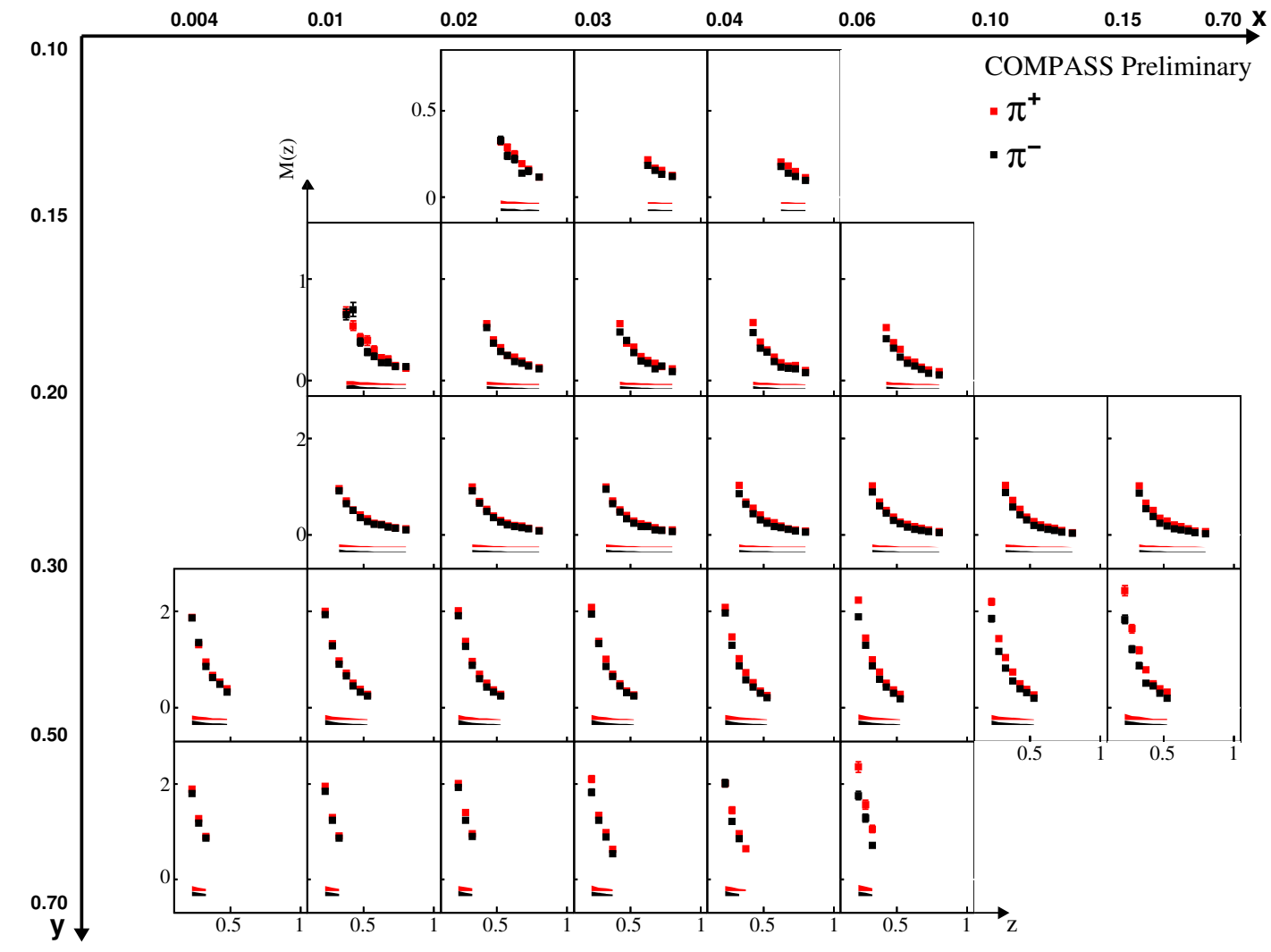

Figure 2: The preliminary, corrected pion hadron multiplicities in bins of $x, y$ and $z$ for positive pions (red) and negative pions (black). The systematic uncertainties are shown as error bands.

the identification efficiency for pions is $98 \%$. The systematic uncertainty reaches from $1 \%$ to $3 \%$.

\section{Result}

Figure 1 and Figure 2 show the preliminary results for unidentified hadrons and pion multiplicities for both charges: red (gray) for positive and black for negative hadrons. The systematic uncertainties are shown as bands in the corresponding $x, y$ and $z$ bins. The unidentified hadron multiplicities and the pion multiplicity are very similar since $70 \%$ of the produced hadrons are in fact pions. The multiplicities show a strong dependence on the hadron energy fraction $z$ and a small dependence on $y$. The dependence on $x$ is rather small and is due to the parton distribution functions. A small difference between positive and negative hadrons is observed in the pion case. With higher $x$, the positive pion multiplicities are larger than the negative ones. The unidentified hadron multiplicities where also the kaons are included have a stronger asymmetry between positive and negative hadrons. Besides acceptance correction and radiative effects, further corrections have to be taken into account: a correction for electron contamination as well as a contribution of vector mesons. 


\section{Additional Corrections}

Lepton-nucleon scattering can result in diffractively produced vector mesons which decay into lighter mesons ( $\pi$ or K). The dominant channel at COMPASS kinematics is the diffractive production of $\rho^{0}$ either produced elastically $\gamma^{*} p \rightarrow \rho^{0} p \rightarrow p \pi^{+} \pi^{-}$or by the decay of an excited target nucleon: $\gamma^{*} p \rightarrow \rho^{0} N^{*}(\Delta) \rightarrow p \pi^{+} \pi^{-}$. Those processes can not directly be distinguished from hadrons produced in SIDIS, thus the multiplicities have to be corrected for this effect. A MonteCarlo study was used to evaluate the contribution of pions coming from the exclusive production of $\rho^{0}$. Two MC data with two different generators were analysed: a SIDIS sample, using LEPTO and a diffractive $\rho^{0}$ sample, using HEPGEN [10], where both, elastic and inelastic processes are considered. Firstly, $\alpha_{\rho^{0}}^{\pi^{ \pm}}$, the fraction of $\pi^{ \pm}$resulting from a diffractive $\rho^{0}$ is determined. Secondly, $\beta_{\rho^{0}}^{D I S}$, the fraction of diffractive $\rho^{0}$ events counted as DIS events is evaluated. In combination, $\alpha_{\rho^{0}}^{\pi^{ \pm}}$ and $\beta_{\rho^{0}}^{D I S}$ lead to the multiplicative correction factor to the multiplicities. The corrections reaches up to $40 \%$ at low $Q^{2}$ and high $z$ in a region, where the values of the multiplicities are very small. In the higher $Q^{2}$ region, the correction is only a few percent.

Electrons within the analysed momentum range (10 to $40 \mathrm{GeV} / \mathrm{c}$ ) can accidentally be mistaken as pions and enlarge the multiplicities. To estimate this yield, real data and the Monte-Carlo data (LEPTO) were compared to extract a correction factor for the multiplicities. The electron contamination factor was estimated in the momentum regions between 3 and $8 \mathrm{Gev}$, where the RICH detector is able to distinguish pions and electrons. The electron yield between real data and MC data is compared: the MC describes well the contamination.Thus, the Monte-Carlo is used to determine the correction in the momentum range $10-40 \mathrm{Gev} / \mathrm{c}$. The correction is $5 \%$ for low $\mathrm{z}$ and smaller than $1 \%$ for high $\mathrm{z}$.

\section{Fragmentation Function}

The fragmentation functions are extracted with a fit to the obtained experimental multiplicities. Firstly, the fragmentation functions are parametrised at a given $Q_{0}^{2}$ and then evolved using the DGLAP equations [11] to the $Q^{2}$ of the given data point. Using the charge and isospin symmetry on Eq. (1.1), two independent fragmentation functions, the so called favoured and unfavoured fragmentation function in combination with the parton distribution functions remain. A $\chi^{2}$ fit is done using the functional form $z D_{\text {fav }}=z D_{\text {unfav }}=\mathscr{N} z^{\alpha}(1-z)^{\beta}\left[1+\gamma(1-z)^{\delta}\right]$ where $\mathscr{N}$ is the normalisation and $\alpha, \beta$ and $\gamma$ are fit parameters. To estimate uncertainties, the fit is performed serveral times with randomly modified experimental multiplicities. The randomised multiplicities are Gaussian distributed with the center and standard deviation corresponding to the experimental multiplicity values and errors respectively. Figure 3 shows the result of the favoured and unfavoured fragmentation functions $z D$ as a function of $z$. In the same figure, results of fragmentation functions from HKNS [12] and DSS [5] are shown. The COMPASS result agrees well with the parametrisation coming from DSS. 

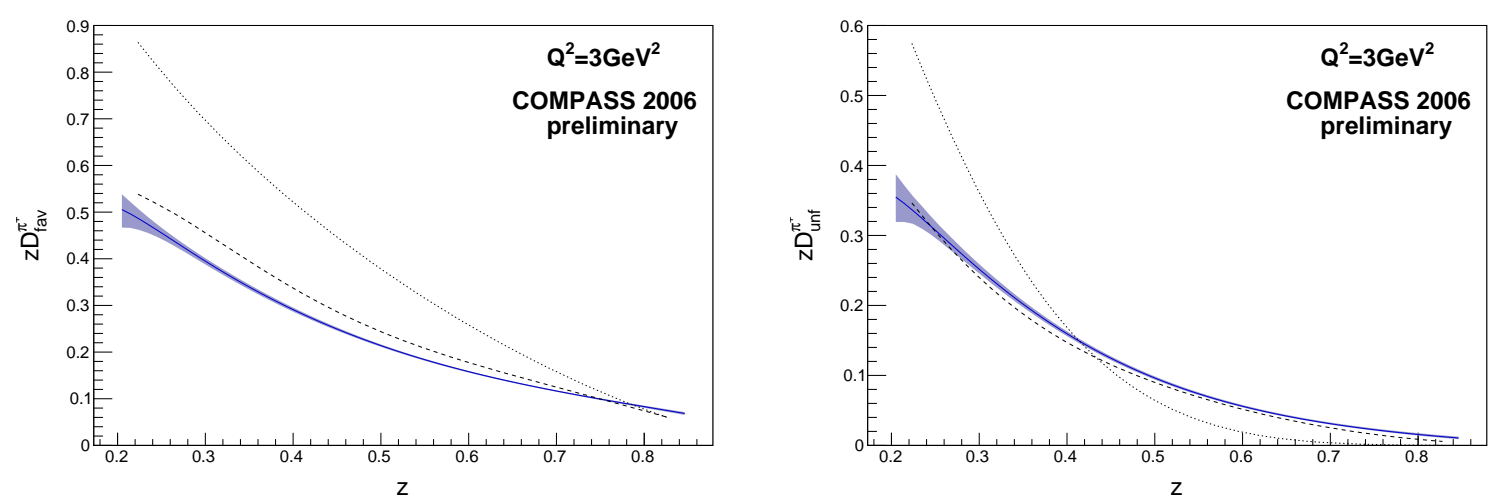

Figure 3: Favoured (left) and unfavoured (right) fragmentation function $z D$ as a function of $z$ with error band coming from the fit error with the result of HKNS (black line) and DSS (dashed line).

\section{Summary and Outlook}

In conclusion, COMPASS has measured the hadron multiplicities for pions and unidentified hadrons from deep inelastic scattering off an isoscalar target with a systematic uncertainty up to $6 \%$, depending on the kinematic range. The preliminary result for the COMPASS fits of fragmentation function have been shown and they agree well with the paramertisation of DSS. Two correction factors have been presented, the correction for diffractively produced $\rho^{0}$ and the contamination of electrons in the pion sample. Currently, the determination of $\mathrm{K}_{S}^{0}$ and $\mathrm{K}^{ \pm}$multiplicities are ongoing, where soon results are expected. In 2012, COMPASS took data with an unpolarised liquid hydrogen target. More data will be taken in the future, most likely after 2015. The large data sample will allow the measurement of hadron multiplicities $M^{h}\left(x, y, z, p_{t}, \phi_{h}\right)$ with the hadron transverse momentum $p_{t}$ and the hadron azimuthal angle $\phi$.

\section{References}

[1] G. Altarelli, Phys. Rept. 81 (1982) 1

[2] V.Yu. Alexakhin, Phys. Lett. B647 (2007) 8

[3] M.G. Alekseev, Phys. Lett. B693 (2010) 227

[4] M. Arneodo, Nucl. Phys. B321 (1989) 541

[5] D. de Florian, Phys. Rev. D75 (2007) 114010

[6] P. Abbon, Nucl. Instr. and Methods A577 (2007) 455

[7] G. Ingelman, Comput.Phys.Commun. 101 (1997) 108

[8] T. Sjöstrand, Comp. Phys. Comm. 82 (1994) 74

[9] C. Adolph, Phys. Lett. B82 (2013) 922

[10] A. Sandacz, arXiv:1207.0333 [hep-ph]

[11] G. Altarelli, Nucl.Phys. B126 (1977) 298

[12] Hirai et al., Phys. Rev. D75 (2007) 094009 